\title{
Clinicall Investigations
}

\section{Development and Validation of a Noninvasive Method to Estimate Cardiac Output Using Cuff Sphygmomanometry}

\author{
Shiu-Shin Chio Ph.D., ${ }^{*}$ Jeffrey J. Tsai, M.S., ${ }^{*}$ Yen-Ming Hsu, M.S., ${ }^{*}$ Jeffrey C. Lapointe, B.S., ${ }^{*}$ Thao Huynh-Covey, \\ R.D.C.S., ${ }^{\dagger}$ Oi Ling B. Kwan, R.D.C.S., ${ }^{\dagger}$ Anthony N. DeMaria, M.D., F.A.C.C. ${ }^{\dagger}$ \\ ${ }^{*}$ Pulse Metric, Inc.; ${ }^{\dagger}$ University of California, San Diego Medical Center-Hillcrest, San Diego, California, USA.
}

\section{Summary}

Background: Obtaining cardiac output (CO) measurements noninvasively during routine blood pressure recording can improve hypertension management. A new method has been developed that estimates cardiac output using pulse-waveform analysis (PWA) from a brachial cuff sphygmomanometer. This study evaluates the ability of PWA to track changes in $\mathrm{CO}$ as derived by Doppler ultrasound during dobutamine stimulation.

Hypothesis: This study aims to validate the PWA CO estimation over a wide $\mathrm{CO}$ range as would be obtained by dobutamine stimulation during Doppler ultrasound evaluation.

Method: A total of 48 patients undergoing standard dobutamine stress echocardiography testing for accepted clinical indications were enrolled. Among them, 44 patients (age 36-83, 18 females, 26 males) with good waveform data for analyses provided estimates of $\mathrm{CO}$ in this study. Noninvasive measurements of $\mathrm{CO}$ were performed using both Doppler ultrasound recordings and PWA techniques simultaneously at each stage of dobutamine infusion.

Results: A total of 207 simultaneous pulse-waveform analyses and Doppler measurements were taken during dobutamine stress on 44 cardiac patients. Linear

Address for reprints:

Anthony N. DeMaria, M.D.

Cardiovascular Division

UCSD Medical Center

200 West Arbor Street

San Diego, CA 92103, USA

e-mail: ademaria@ucsd.edu

Received: December 19, 2006

Accepted: April 7, 2007

Published online in Wiley InterScience

(www.interscience.wiley.com).

DOI: $10.1002 / \mathrm{clc} .20164$

(C) 2007 Wiley Periodicals, Inc. regression analysis revealed good intra-patient correlation between pulse-waveform analysis and Doppler at different dobutamine-induced $\mathrm{CO}$ with coefficients from $\mathrm{r}=0.69$ to $0.98(p<0.05)$. Analysis of all patients yielded an overall correlation of $\mathrm{r}=0.82 \quad(p<0.001$, bias $=0.4 \mathrm{~L} / \mathrm{min}$, standard deviation $=1.8 \mathrm{~L} / \mathrm{min}$ ).

Conclusion: The $\mathrm{CO}$ measured noninvasively from a sphygmomanometer using this PWA method correlates well with those of Doppler through a range of dobutamine-stimulated levels. The CO by PWA should be useful for monitoring hemodynamic changes in hypertensive and cardiac patients during routine blood pressure measurement.

Key words: cardiac output, pulse-waveform analysis, thermo-dilution, Doppler ultrasound, dobutamine stimulation, hypertension

Clin. Cardiol. 2007; 30: 615-620.

(C) 2007 Wiley Periodicals, Inc.

\section{Introduction}

Recent data suggests that hemodynamic approaches to the management of hypertension may be superior to standard clinical care, using additional treatment algorithms based on cardiac output (CO) and systemic vascular resistance. ${ }^{1}$ Anti-hypertensive therapies should not only lower arterial blood pressure but also improve hemodynamics and normalize functional and structural components of the cardiovascular system. ${ }^{2}$ On the basis of cardiac waveform analysis methods, ${ }^{3-12}$ newer noninvasive devices that measure various hemodynamic parameters other than blood pressure, including $\mathrm{CO}$ and arterial compliance have recently become available. A simple noninvasive technique has been developed to estimate $\mathrm{CO}$ based on arterial PWA method. Combining this PWA $\mathrm{CO}$ to our previously developed Pulse-Dynamics (PD) 
Method $^{11-13}$ that measures blood pressures, heart rate (HR), arterial compliance and resistance conveys the potential to provide hemodynamic profiles during routine sphygmomanometry. This PD technology analyzes the oscillometric brachial arterial pressure waveform, Figure 1, acquired from the cuff sphygmomanometer to obtain the rate of pressure change $\frac{d P}{d t}$, systolic blood pressure (SBP) and diastolic blood pressure (DBP) as illustrated in Figure 2.

In the present study, we have described and derived estimates of CO by this PWA PD CO methodology, and compared them with those from Doppler ultrasound (echo) method at various levels of $\mathrm{CO}$ occurring during dobutamine stress echocardiography. ${ }^{14}$

\section{Methods}

Study Group: The study group consisted of 48 patients undergoing standard dobutamine stress echocardiography testing for accepted clinical indications at the University of California, San Diego (UCSD) Medical Center. Three patients were excluded due to severe motion artifacts and irregular heart beats throughout the examination. One additional patient was excluded due to extreme obesity and the blood pressure cuff not fitting the upper arm properly. The remaining 44 patients provided estimates of $\mathrm{CO}$ in this study. The group consisted of 26 males and 18 females, age 36-83 (mean \pm SD of $65 \pm 12$ years), and of varying races ( 31 caucasian, 7 blacks, 3 Hispanic, 2 Asian, and 1 Middle Eastern). None of the patients exhibited any clinical condition (such as significant valvular regurgitation or severe arrhythmias), which would alter Doppler CO measurements. Patients gave informed consent and the study protocol was approved by the Institutional Review Board.

\section{Dobutamine Stress}

The primary indication for the dobutamine stress echocardiography protocol used in this study was to evaluate the presence of coronary artery disease. The betaadrenergic agonist effects of dobutamine hydrochloride increase myocardial contractility and heart rate (HR), thus increasing CO. Throughout the examination, electrocardiogram (ECG) and blood pressure measurements were continuously monitored, while intravenous dobutamine was administered in increasing concentration at intervals of $10 \mathrm{mcg} / \mathrm{kg} / \mathrm{min}$ from 0 to $40 \mathrm{mcg} / \mathrm{kg} / \mathrm{min}$. Atropine in doses of 0.5 to $1.0 \mathrm{mg}$ was administered to $65 \%$ of the patients in whom the HR increase in response to dobutamine was inadequate. At each stage of dobutamine infusion the sonographer captured echocardiographic images to assess ventricular wall thickening and endocardial excursion. The study ended when the patient manifested myocardial ischemia or arrhythmias, or reached the target HR of $85 \%$ of maximum HR calculated using an age-based scale.

\section{Procedures}

Noninvasive measurements of $\mathrm{CO}$ were performed using both Doppler ultrasound recordings and PD (DynaPulse 200M, Pulse Metric, Inc., San Diego, Calif.) techniques simultaneously at each stage of dobutamine infusion. During recovery, measurements were obtained at every HR decrease of $10-15$ beats per minute until the examination was concluded. The original data recorded by the DynaPulse device was first stored on a PC and then uploaded to a server (DynaPulse Analysis Center $[\mathrm{DAC}]$ ) where the arterial pulsation signal data was processed using proprietary algorithms. The Doppler echocardiographic signals were recorded on paper for subsequent analysis and measurement.

The procedure for simultaneously capturing $\mathrm{CO}$ measurements involved direct coordination between the sonographer and the technician acquiring arterial recordings. The left ventricular outflow tract (LVOT) diameter was first obtained. Then, as the blood pressure cuff deflated to record the pulsating oscillometric signal, the sonographer began acquiring the flow velocity profile at the LVOT. When the cuff deflated to DBP, as judged by the graphic display of a mercury sphygmomanometer, the sonographer captured and freeze-framed the velocity profile, traced the profile to calculate the systolic velocity time integral, and used that information to determine the stroke volume of that specific cardiac cycle. This procedure was established to ensure proper timing and accuracy of the $\mathrm{CO}$ measurements, since stroke volume may change substantially from beat to beat.

\section{Measurement Techniques}

Echo method: Measurements of CO by Doppler ultrasound were calculated as the product of the systolic flow velocity integral (FVI) and the cross-sectional area (CSA) of the LVOT. The largest diameter of the LVOT was identified by medial/lateral scanning in the parasternal long axis view at a level just below the aortic valve annulus. The cross-sectional area of the LVOT was then obtained as $\pi\left(\frac{\text { diameter }}{2}\right){ }^{2}$ The flow velocity signal in the LVOT was obtained with a pulsed Doppler sample volume positioned at the aortic annulus. The time velocity integral of LVOT flow was derived internally within the echograph from an operator trace of modal velocity during systole. $\mathrm{CO}$ was calculated as $F V I * C S A$.

DynaPulse method: The PD technique was used to determine SBP, DBP and mean arterial pressure (MAP) by PWA of the oscillometric cuff signal (Figure 2) from the brachial artery. ${ }^{12,13,15}$ The PWA PD CO is then derived by further analyzing the changes in PD pressure waveform. First, BA $\frac{d P}{d t}$ max obtained at brachial artery (Figure 2) was used to approximate left ventricular $(\mathrm{LV}) \frac{d P}{d t} \max _{\max }$ and LV contractility (LVC) using Gaussian 


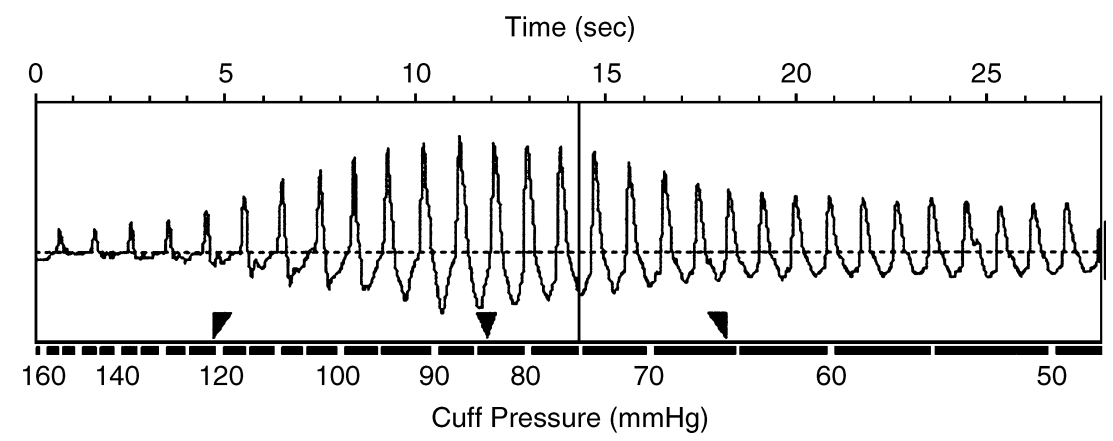

FIG. 1 Oscillometric pulse-dynamics waveform.

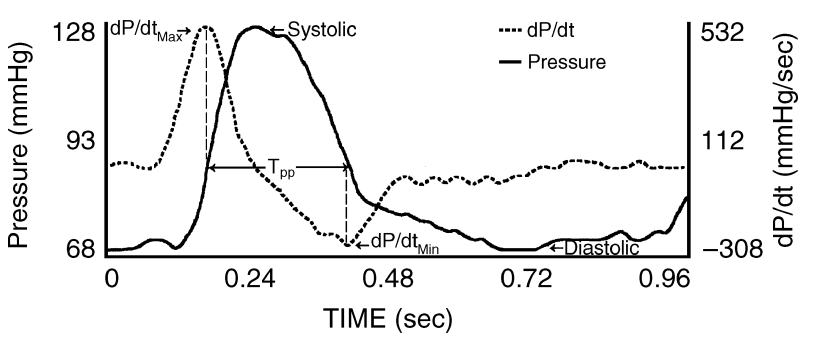

FIG. 2 A Pulse-Dynamics arterial pressure waveform (normalized to SBP and DBP).

transformation function, ${ }^{16-18}$ shown below:

$$
\begin{aligned}
L V \frac{d P}{d t}_{\max } & =\frac{B A \frac{d P}{d t} \max }{T r} * e^{\frac{T r^{2}-1}{2}}, \text { and } \\
L V C & =\frac{B A \frac{d P}{d t} \max }{T r} * \frac{e^{\frac{T r^{2}}{2}}}{S B P}
\end{aligned}
$$

where,

$$
\frac{1}{T r}=\frac{T p p_{L V}}{T p p_{B A}}=\sqrt{1+\frac{D B P}{S B P-D B P} * \sqrt{e}}, \text { and }
$$

$T p p=$ time interval between $\frac{d P}{d t}$ max to $\frac{d P}{d t}$ min $_{\text {for }}$ BA and LV pressure contours.

The $\mathrm{CO}$ was then obtained using equation $C O \propto$ $L V C * H R * B S A$ and an empirically determined scaling factor obtained by comparing to thermo-dilution (TD) CO of 11 non-PH patients from a previous study ${ }^{12}$ (data shown in Table 1). Where, body surface area (BSA) is defined by standard DuBois equation, a function of weight and height.

\section{Statistics}

Statistical analyses were performed using Microsoft Excel (Redmond, Wash., USA) and SPSS (SPSS, Inc., Chicago, Ill., USA). Results were expressed using linear

\begin{tabular}{|c|c|c|c|c|c|}
\hline $\begin{array}{l}\text { Patient } \\
\text { group }\end{array}$ & $\mathrm{r}$ & $\mathrm{p}$ & $\mathrm{N}$ & $\begin{array}{c}\text { Bias } \\
(\mathrm{L} / \mathrm{min})\end{array}$ & $\begin{array}{c}\text { Standard } \\
\text { deviation } \\
(\mathrm{L} / \mathrm{min})\end{array}$ \\
\hline PH patients ${ }^{a}$ & 0.81 & $<0.01$ & z09 & -1.8 & 0.7 \\
\hline $\begin{array}{l}\text { Non-PH } \\
\text { patients }\end{array}$ & 0.63 & $<0.04$ & 11 & -0.0 & 0.8 \\
\hline All patients & 0.47 & $<0.04$ & 20 & -0.8 & 1.1 \\
\hline
\end{tabular}
regression analysis, showing the correlation coefficient
TABLE 1 Summary of statistics of PD CO versus TD CO

${ }^{a} \mathrm{PH}=$ pulmonary hypertension.

and the significance of correlation between the two methods. A Bland-Altman analysis was performed to show the precision and bias between the two techniques. ${ }^{19}$

Multivariate analysis was also performed using stepwise linear regression on selected variables to determine the independent predictors of CO measured by Doppler ultrasound. Similar analyses were performed with PD $\mathrm{CO}$ as a model comparison.

\section{Results}

The protocol was successfully completed in 44 patients. No severe arrhythmias or adverse events were experienced. Technically adequate data for analysis was obtained in all patients. The HR ranged from $44-139 \mathrm{bpm}$ and Doppler CO from 2.91 to $21.70 \mathrm{~L} / \mathrm{min}$. Blood pressures ranged from $97-225 \mathrm{mmHg}$ systolic, and $42-116 \mathrm{mmHg}$ diastolic. A total of 207 simultaneous measurements of CO by Doppler and PD were available for statistical analysis.

Figure 3 displays results of linear regression analysis comparing all 207 Doppler and PD measurements. The analysis yielded a relation of $y=0.74 x+2.42$ between Doppler ultrasound and PD values of CO. There was a small systematic overestimation of Doppler CO by PD $\mathrm{CO}$. Linear regression analysis revealed a good correlation, $r=0.82, p<0.001$. Figure 4 illustrates the results of Bland-Altman analysis of the data. The dispersion of values can be seen to increase at $\mathrm{CO}$ levels above 6.0 $\mathrm{L} / \mathrm{min}$ with an overall standard deviation of $1.8 \mathrm{~L} / \mathrm{min}$. 


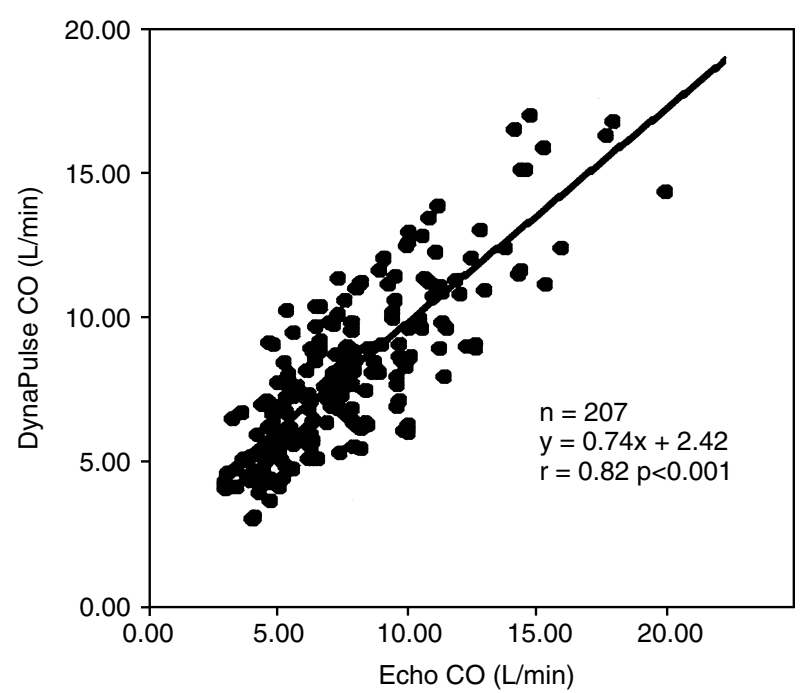

FIG. 3 Linear regression analysis of DynaPulse PD CO versus Doppler echo CO.

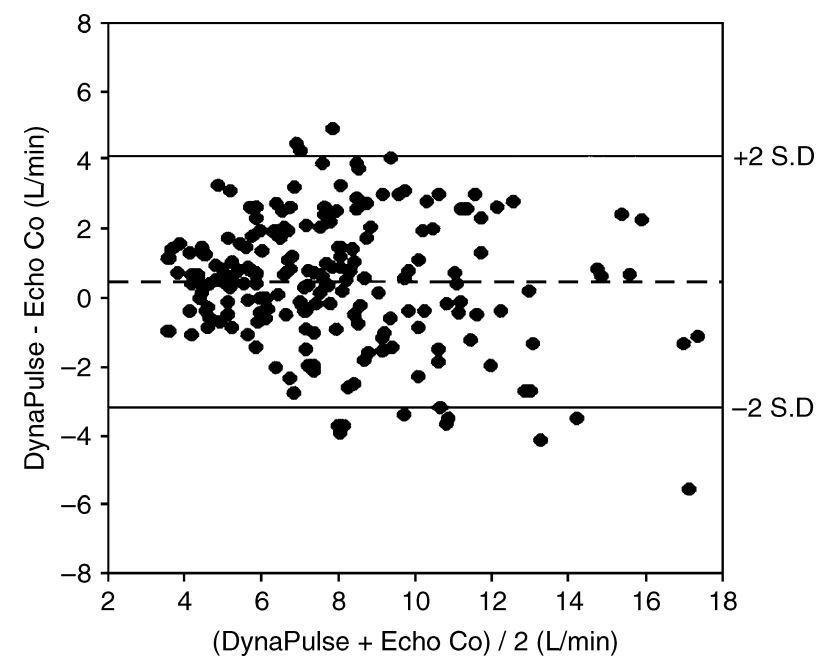

FIG. 4 Bland-Altman analysis comparing DynaPulse PD CO versus Doppler echo CO.

Table 2 displays the correlation between Doppler and PD CO for each stage of the dobutamine protocol. The wider ranges of $\mathrm{CO}$ encountered during infusion and recovery yielded greater correlation coefficients than were observed at baseline. Nevertheless, the bias and precision values were generally similar for all three stages. Overall correlation coefficient was very significant $(\mathrm{r}=0.82, \mathrm{p}<0.001, \mathrm{n}=207)$.

Table 3 shows partial correlation coefficients in multivariate analysis to determine the significant independent predictors of Doppler CO. The model included age, height, weight, SBP, DBP, MAP, pulse pressure, HR, and derived LVC. The significant predictors were HR, weight, LVC, height, and DBP. The overall correlation coefficient for the model was $r=0.83$. Table 4 shows
TABLE 2 Summary of statistics (PD CO versus Doppler $\mathrm{CO})$ at each stage dobutamine stress

\begin{tabular}{lccrrc}
\hline \multicolumn{1}{c}{ Stage } & $\mathrm{r}$ & $\mathrm{p}$ & $\mathrm{n}$ & $\begin{array}{c}\text { Bias } \\
(\mathrm{L} / \mathrm{min})\end{array}$ & $\begin{array}{c}\text { Standard } \\
\text { deviation } \\
(\mathrm{L} / \mathrm{min})\end{array}$ \\
\hline Baseline & 0.70 & $<0.001$ & 36 & 0.33 & 1.64 \\
Infusion & 0.70 & $<0.001$ & 101 & -0.13 & 2.60 \\
Recovery & 0.77 & $<0.001$ & 77 & 0.68 & 1.95 \\
Overall & 0.82 & $<0.001$ & 207 & 0.45 & 1.83 \\
\hline
\end{tabular}

TABLE 3 Multivariate analysis for significant predictors of Doppler CO

\begin{tabular}{lcr}
\hline \multicolumn{1}{c}{ Variable entered $^{a}$} & Partial correlation & \multicolumn{1}{c}{$\mathrm{p}$} \\
\hline HR & 0.695 & $<0.001$ \\
Weight & 0.572 & $<0.001$ \\
LVC & 0.492 & $<0.001$ \\
Height & -0.254 & 0.008 \\
DBP & -0.217 & 0.023 \\
\hline
\end{tabular}

Overall model $\mathrm{r}=0.834$.

${ }^{a}$ Model included Height, Weight, SBP, DBP, MAP, PP, HR, LVC (contractility).

the results of the same analysis performed against PD $\mathrm{CO}$. The significant determinants were HR, weight, LVC, SBP, and height. The overall model correlation was $\mathrm{r}=$ 0.98 with $\mathrm{p}<0.001$.

\section{Discussion}

Considerable effort continues to be directed towards implementing noninvasive methods to quantify $\mathrm{CO}$. The recent development of PD technology to acquire an oscillometric pressure waveform has provided a potential to estimate CO. The results of this study show good correlations between $\mathrm{CO}$ derived from PD and Doppler ultrasound techniques during dobutamine stress echocardiography. The correlation was very good during stressinduced augmentation of $\mathrm{CO}$, and showed lesser but acceptable correlations during baseline measurements. Thus, this new technique offers the potential to obtain quantitative estimates of $\mathrm{CO}$, particularly with regard to determination of directional changes, in the course of measuring blood pressure by cuff sphygmomanometry.

\section{Previous Study}

In the development of this new PWA PD CO estimating method, we compared PD CO to TD CO from a previous study, to obtain the scaling factor, twenty patients, 17 men, aged 46-78 years, underwent rightheart catheterization at UCSD Medical Center, while their TD CO and PD blood pressure and pulse waveform 
TABLE 4 Multivariate analysis for significant predictors of PD CO

\begin{tabular}{lcc}
\hline \multicolumn{1}{c}{ Variable entered $^{a}$} & Partial correlation & $\mathrm{p}$ \\
\hline HR & 0.941 & $<0.001$ \\
Weight & 0.918 & $<0.001$ \\
LVC & 0.897 & $<0.001$ \\
SBP & -0.814 & $<0.001$ \\
Height & -0.514 & $<0.001$ \\
\hline
\end{tabular}

Overall model $\mathrm{r}=0.983$.

aModel included height, weight, SBP, DBP, MAP, PP, HR, LVC (contractility).

were obtained as described in an earlier paper. ${ }^{12}$ Among the 20 patients, 9 had been diagnosed with pulmonary hypertension $(\mathrm{PH})$ and only the remaining 11 non-PH patients were used to calculate the scaling factor for the PD CO estimation. Table 1 exhibits PD CO values (average of 3 corresponding PD waveform analyses) estimated by this PWA method and compared to the TD CO (average of 3 determinations) for $9 \mathrm{PH}$ and 11 non-PH cases and all 20 patients. In this study, we observed that TD $\mathrm{CO}$ in the PH group was significantly lower (mean 3.62 $\mathrm{L} / \mathrm{min}$ ) than non-PH group (mean $4.84 \mathrm{~L} / \mathrm{min}$ ). Measuring $\mathrm{CO}$ in $\mathrm{PH}$ patients may introduce unknown variables. We also chose $\mathrm{CO}$ data from the non-PH group to obtain the scaling factor, since no $\mathrm{PH}$ patient was admitted in the Echo-dobutamine stress study.

\section{Limitations}

The PD technique derives CO primarily from LVC, BSA and HR, and Doppler CO measurement is based on LVOT flow and cross-section area. Multivariate analysis showed similarities between the independent predictors of Doppler CO and the determinants of PD CO. In both methods, HR, weight, and LV contractility were significant contributors to the model. The SBP was a significant determinant for PD CO but not for Doppler CO, while DBP was significant for Doppler CO but not for PD CO. Further refinements of PD account for these variables may be forthcoming. When comparing with invasive TD $\mathrm{CO}$ measurement, noninvasive $\mathrm{PD}$ $\mathrm{CO}$ was found to overestimate $\mathrm{PH}$ patients since it was normalized to non-PH TD CO group. This is a limitation of the current PD CO technique, unless a prediagnosis of $\mathrm{PH}$ is made available and a different scaling factor is used in PD CO calculation.

Other limitations may prevent the application of PD $\mathrm{CO}$ estimation in specific disease populations. Patients with severe obesity (BMI $\geqslant 40 \mathrm{~kg} / \mathrm{m}^{2}$ ) may exhibit abnormal nonlinear characteristics resulting in exceptions to the physical model used, possibly excluding this population from the PD application. The oscillometric cuff-based PD technology also has inherent weaknesses due to artifacts of brachial artery pressure waveform.
Artifacts may include both intrinsic sources including severe arrhythmias, valvular disorders, or vascular interventions, and extrinsic sources such as motion artifacts. Severe pulse waveform artifacts that could not be filtered by DynaPulse hardware and software may limit the technology in some clinical applications. Evaluation of specific waveform morphology should be considered during physician assessment in these cases. Despite these limitations, the ability to monitor changing values of $\mathrm{CO}$ as well as to estimate systemic vascular resistance (SVR), as derived by $S V R=\frac{M A P}{C O}$, would be useful in monitoring the overall hemodynamic status of patients with hypertension or cardiovascular diseases.

It should be noted that echocardiographic assessment of $\mathrm{CO}$ also has its limitations. The method assumes that the LVOT is circular and does not change in size or shape through the cardiac cycle, that the flow velocity profile is uniform across the vessel, that the ultrasound beam is parallel to the direction of flow, and that the diameter of the flow channel can be accurately measured. These assumptions may limit the precision with which $\mathrm{CO}$ and SV can be made by Doppler ultrasound, and may have influenced the results of this study.

\section{Conclusions}

We have developed and described a novel noninvasive method to derive estimates of $\mathrm{CO}$ from the same oscillometric technology used to determine arterial pressure. Our results indicate that this method can provide estimates of $\mathrm{CO}$ that correlate with those obtained by echocardiography. The directional changes seen in PD $\mathrm{CO}$, as evidenced by the ability to accurately detect alterations induced by dobutamine can be of added value in the ability to trend changes in cardiovascular function. This additional data, simply and noninvasively acquired while measuring routine blood pressure, has the potential to add clinically valuable information that may be used in managing patients with hypertension and other cardiovascular conditions.

\section{Acknowledgements}

We thank the University of California, San Diego Medical Center, that provided facilities, and its staff at the catheterization and Echo laboratories for assistance in data collection. In addition, we acknowledge the support of Pulse Metric, Inc. that provided DynaPulse-200M devices and the access to its DAC for online PulseDynamics cardiac output analysis, and support of its staff.

\section{References}

1. Taler SJ, Textor SC, Augustine JE: Resistant hypertension: comparing hemodynamic management to specialist care. Hypertension 2002;39:982-988 
2. Messerli FH: Calcium antagonists in hypertension: from hemodynamics to outcomes. Am J Hypertens 2002;15:94S-97S

3. Shoemaker WC, Wo CJ, Bishop MH, Appel PL, van de Water JM, et al.: Multicenter trial of a new thoracic electrical bioimpendance device for cardiac output estimation. Crit Care Med 1994;22:1907-1912

4. Hirschl MM, Kittler H, Woisetschager C, Siostrzonek P, Staudinger $\mathrm{T}$, et al:: Simultaneous comparison of thoracic bioimpedance and arterial pulse waveform-derived cardiac output with thermo-dilution measurement. Crit Care Med 2000;28:1798-1802

5. Hirschl MM, Binder M, Gwechenberger M, Herkner H, Bur A, et al. Non-invasive assessment of cardiac output in critically ill patients by analysis of the finger blood pressure waveform. Crit Care Med 1997;25:1909-1914

6. Liu Z, Brin KP, Yin FC: Estimation of total arterial compliance: an improved method and evaluation of current methods. Am J Phisol 1986;251:H588-H600

7. McVeigh GE, Burns DE, Finkelstein SM, McDonald KM, Mock JE, et al.: Reduced vascular compliance as a marker for essential hypertension. Am J Hypertens 1991;4:245-251

8. O'Rourke MF, Brunner HR: Introduction to arterial compliance and function. J Hypertens 1992;10:S3-S5

9. Watt TB, Burrus CS: Arterial pressure contour analysis for estimating human vascular properties. J Appl Physiol 1976;40: $171-176$

10. Simon AC, Safar ME, Levenson JA, London GM, Levy BI, et al. An evaluation of large arteries compliance in man. Am J Physiol 1979;237:H550-H554
11. Brinton TJ, Kailasam MT, Wu RA, Cervenka JH, Chio SS, et al.: Arterial compliance by cuff sphygmomanometer. Application to hypertension and early changes in subjects at genetic risk. Hypertension 1996;28:599-603

12. Brinton TJ, Cotter B, Kailasam MT, Brown DL, Chio SS, et al.: Development and validation of a noninvasive method to determine arterial pressure and vascular compliance. Am J Cardiol 1997;80:323-330

13. Brinton TJ, Walls ED, Chio SS: Validation of pulse dynamic blood pressure measurement by auscultation. Blood Press Monit 1998;3:121-124

14. Armstrong WF, Zoghbi WA: Stress echocardiography current methodology and clinical applications. $J$ Am Coll Cardiol 2005; $45: 1739-1747$

15. Chio SS. Method and apparatus for determining blood pressure and cardiovascular condition. U.S. Patent 4,880,013. November 14, 1989

16. Chio SS. Method for diagnosing, monitoring and treating hypertension and other cardiac problems. U.S. Patent 5,836,884. November 17, 1998

17. Chio SS. Method for diagnosing, monitoring and treating hypertension and other cardiac problems. U.S. Patent 6,165,130. December 26, 2000

18. Chio SS. Method for diagnosing, monitoring and treating hypertension and other cardiac problems. U.S. Patent 6,270,461, Aug. 7, 2001, and U.S. Patent 6,540,687 B2, Apr. 1, 2003

19. Bland JM, Altman DG: Statistical methods for assessing agreement between two methods of clinical measurement. Lancet 1986;1:307-310 